\title{
Asymptotic Behaviour of a Two-Dimensional Differential System with a Finite Number of Nonconstant Delays under the Conditions of Instability
}

\author{
Zdeněk Šmarda ${ }^{1}$ and Josef Rebenda ${ }^{2}$ \\ ${ }^{1}$ Department of Mathematics, Faculty of Electrical Engineering and Communication, \\ Brno University of Technology, 61600 Brno, Czech Republic \\ ${ }^{2}$ Department of Mathematics and Statistics, Faculty of Science, Masaryk University, \\ 61137 Brno, Czech Republic
}

Correspondence should be addressed to Zdeněk Šmarda, smarda@feec.vutbr.cz

Received 20 February 2012; Accepted 4 April 2012

Academic Editor: Miroslava Růžičková

Copyright (C) 2012 Z. Šmarda and J. Rebenda. This is an open access article distributed under the Creative Commons Attribution License, which permits unrestricted use, distribution, and reproduction in any medium, provided the original work is properly cited.

\begin{abstract}
The asymptotic behaviour of a real two-dimensional differential system $x^{\prime}(t)=\mathrm{A}(t) x(t)+$ $\sum_{k=1}^{m} \mathrm{~B}_{k}(t) x\left(\theta_{k}(t)\right)+h\left(t, x(t), x\left(\theta_{1}(t)\right), \ldots, x\left(\theta_{m}(t)\right)\right)$ with unbounded nonconstant delays $t-\theta_{k}(t) \geq$ 0 satisfying $\lim _{t \rightarrow \infty} \theta_{k}(t)=\infty$ is studied under the assumption of instability. Here, $A, B_{k}$, and $h$ are supposed to be matrix functions and a vector function. The conditions for the instable properties of solutions and the conditions for the existence of bounded solutions are given. The methods are based on the transformation of the considered real system to one equation with complex-valued coefficients. Asymptotic properties are studied by means of a Lyapunov-Krasovskii functional and the suitable Ważewski topological principle. The results generalize some previous ones, where the asymptotic properties for two-dimensional systems with one constant or nonconstant delay were studied.
\end{abstract}

\section{Introduction}

Consider the real two-dimensional system

$$
x^{\prime}(t)=\mathrm{A}(t) x(t)+\sum_{k=1}^{m} \mathrm{~B}_{k}(t) x\left(\theta_{k}(t)\right)+h\left(t, x(t), x\left(\theta_{1}(t)\right), \ldots, x\left(\theta_{m}(t)\right)\right),
$$


where $\theta_{k}(t)$ are real functions, $\mathrm{A}(t)=\left(a_{i j}(t)\right), \mathrm{B}_{k}(t)=\left(b_{i j k}(t)\right)(i, j=1,2 ; k=1, \ldots, m)$ are real square matrices, and $h(t, x, y)=\left(h_{1}\left(t, x, y_{1}, \ldots, y_{m}\right), h_{2}\left(t, x, y_{1}, \ldots, y_{m}\right)\right)$ is a real vector function, $x=\left(x_{1}, x_{2}\right), y_{k}=\left(y_{1 k}, y_{2 k}\right)$. It is supposed that the functions $\theta_{k}, a_{i j}$ are locally absolutely continuous on $\left[t_{0}, \infty\right), b_{i j k}$ are locally Lebesgue integrable on $\left[t_{0}, \infty\right)$, and the function $h$ satisfies Carathéodory conditions on $\left[t_{0}, \infty\right) \times \mathbb{R}^{2(m+1)}$.

There are a lot of papers dealing with the stability and asymptotic behaviour of $n$ dimensional real vector equations with delay. Among others we should mention the recent results [1-13]. Since the plane has special topological properties different from those of $n$ dimensional space, where $n \geq 3$ or $n=1$, it is interesting to study the asymptotic behaviour of two-dimensional systems by using tools that are typical and effective for two-dimensional systems. The convenient tool is the combination of the method of complexification and the method of Lyapunov-Krasovskii functional. For the case of instability, it is useful to add to this combination the version of Ważewski topological principle formulated by Rybakowski in the papers $[14,15]$. Using these techniques, we obtain new and easy applicable results on stability, asymptotic stability, instability, or boundedness of solutions of the system (1.1).

The main idea of the investigation, the combination of the method of complexification and the method of Lyapunov-Krasovskii functional, was introduced for ordinary differential equations in the paper by Ráb and Kalas [16] in 1990. The principle was transferred to differential equations with delay by Kalas and Baráková [17] in 2002. The results in the case of instability were obtained for ODEs by Kalas and Osička [18] in 1994 and for delayed differential equations by Kalas [19] in 2005.

We extend such type of results to differential equations with a finite number of nonconstant delays. We introduce the transformation of the considered real system to one equation with complex-valued coefficients. We present sufficient conditions for the instability of a solution and for the existence of a bounded solution. The applicability of the results is demonstrated with several examples.

At the end of this introduction we append a brief overview of notation used in the paper and the transformation of the real system to one equation with complex-valued coefficients.

$\mathbb{R}$ is the set of all real numbers,

$\mathbb{R}_{+}$the set of all positive real numbers,

$\mathbb{R}_{+}^{0}$ the set of all nonnegative real numbers,

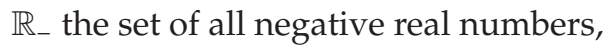

$\mathbb{R}_{-}^{0}$ the set of all nonpositive real numbers,

$\mathbb{C}$ the set of all complex numbers,

$\mathcal{C}$ the class of all continuous functions $[-r, 0] \rightarrow \mathbb{C}$,

$A C_{\text {loc }}(I, M)$ the class of all locally absolutely continuous functions $I \rightarrow M$,

$L_{\text {loc }}(I, M)$ the class of all locally Lebesgue integrable functions $I \rightarrow M$,

$K(I \times \Omega, M)$ the class of all functions $I \times \Omega \rightarrow M$ satisfying Carathéodory conditions on $I \times \Omega$, 
$\operatorname{Re} z$ the real part of $z$,

$\operatorname{Im} z$ the imaginary part of $z$, and

$\bar{z}$ the complex conjugate of $z$.

Introducing complex variables $z=x_{1}+i x_{2}, w_{1}=y_{11}+i y_{12}, \ldots, w_{m}=y_{m 1}+i y_{m 2}$, we can rewrite the system (1.1) into an equivalent equation with complex-valued coefficients

$$
\begin{aligned}
z^{\prime}(t)= & a(t) z(t)+b(t) \bar{z}(t)+\sum_{k=1}^{m}\left[A_{k}(t) z\left(\theta_{k}(t)\right)+B_{k}(t) \bar{z}\left(\theta_{k}(t)\right)\right] \\
& +g\left(t, z(t), z\left(\theta_{1}(t)\right), \ldots, z\left(\theta_{m}(t)\right)\right)
\end{aligned}
$$

where $\theta_{k} \in A C_{\mathrm{loc}}(J, \mathbb{R})$ for $k=1, \ldots, m, A_{k}, B_{k} \in L_{\mathrm{loc}}(J, \mathbb{C}), a, b \in A C_{\mathrm{loc}}(J, \mathbb{C}), g \in K(J \times$ $\left.\mathbb{C}^{m+1}, \mathbb{C}\right), J=\left[t_{0}, \infty\right)$.

The relations between the functions are as follows:

$$
\begin{aligned}
a(t)= & \frac{1}{2}\left(a_{11}(t)+a_{22}(t)\right)+\frac{i}{2}\left(a_{21}(t)-a_{12}(t)\right), \\
b(t)= & \frac{1}{2}\left(a_{11}(t)-a_{22}(t)\right)+\frac{i}{2}\left(a_{21}(t)+a_{12}(t)\right), \\
A_{k}(t)= & \frac{1}{2}\left(b_{11 k}(t)+b_{22 k}(t)\right)+\frac{i}{2}\left(b_{21 k}(t)-b_{12 k}(t)\right), \\
B_{k}(t)= & \frac{1}{2}\left(b_{11 k}(t)-b_{22 k}(t)\right)+\frac{i}{2}\left(b_{21 k}(t)+b_{12 k}(t)\right), \\
g\left(t, z, w_{1}, \ldots, w_{m}\right)= & h_{1}\left(t, \frac{1}{2}(z+\bar{z}), \frac{1}{2 i}(z-\bar{z}), \frac{1}{2}\left(w_{1}+\bar{w}_{1}\right), \ldots, \frac{1}{2 i}\left(w_{m}-\bar{w}_{m}\right)\right) \\
& +i h_{2}\left(t, \frac{1}{2}(z+\bar{z}), \frac{1}{2 i}(z-\bar{z}), \frac{1}{2}\left(w_{1}+\bar{w}_{1}\right), \frac{1}{2 i}\left(w_{1}-\bar{w}_{1}\right), \ldots, \frac{1}{2 i}\left(w_{m}-\bar{w}_{m}\right)\right) .
\end{aligned}
$$

Conversely, putting

$$
\begin{array}{cc}
a_{11}(t)=\operatorname{Re}[a(t)+b(t)], & a_{12}(t)=\operatorname{Im}[b(t)-a(t)], \\
a_{21}(t)=\operatorname{Im}[a(t)+b(t)], & a_{22}(t)=\operatorname{Re}[a(t)-b(t)], \\
b_{11 k}(t)=\operatorname{Re}\left[A_{k}(t)+B_{k}(t)\right], & b_{12 k}(t)=\operatorname{Im}\left[B_{k}(t)-A_{k}(t)\right], \\
b_{21 k}(t)=\operatorname{Im}\left[A_{k}(t)+B_{k}(t)\right], & b_{22 k}(t)=\operatorname{Re}\left[A_{k}(t)-B_{k}(t)\right], \\
h_{1}\left(t, x, y_{1}, \ldots, y_{m}\right)=\operatorname{Reg}\left(t, x_{1}+i x_{2}, y_{11}+i y_{12}, \ldots, y_{m 1}+i y_{m 2}\right), \\
h_{2}\left(t, x, y_{1}, \ldots, y_{m}\right)=\operatorname{Img}\left(t, x_{1}+i x_{2}, y_{11}+i y_{12}, \ldots, y_{m 1}+i y_{m 2}\right),
\end{array}
$$

the equation (1.2) can be written in the real form (1.1) as well. 


\section{Preliminaries}

We consider (1.2) in the case when

$$
\liminf _{t \rightarrow \infty}(|\operatorname{Im} a(t)|-|b(t)|)>0
$$

and study the behavior of solutions of (1.2) under this assumption. This situation corresponds to the case when the equilibrium 0 of the autonomous homogeneous system

$$
x^{\prime}=\mathbf{A} x
$$

where $\mathbf{A}$ is supposed to be regular constant matrix, is a centre or a focus. See [16] for more details.

Regarding (2.1) and since the delay functions $\theta_{k}$ satisfy $\lim _{t \rightarrow \infty} \theta_{k}(t)=\infty$, there are numbers $T_{1} \geq t_{0}, T \geq T_{1}$, and $\mu>0$ such that

$$
|\operatorname{Im} a(t)|>|b(t)|+\mu \quad \text { for } t \geq T_{1}, \quad t \geq \theta_{k}(t) \geq T_{1} \quad \text { for } t \geq T(k=1, \ldots, m) .
$$

Denote

$$
\tilde{\gamma}(t)=\operatorname{Im} a(t)+\sqrt{(\operatorname{Im} a(t))^{2}-|b(t)|^{2}} \operatorname{sgn}(\operatorname{Im} a(t)), \quad \tilde{c}(t)=-i b(t) .
$$

Notice that the above-defined function $\tilde{\gamma}$ need not be positive.

Since $|\tilde{\gamma}(t)|>|\operatorname{Im} a(t)|$ and $|\widetilde{c}(t)|=|b(t)|$, the inequality

$$
|\tilde{\gamma}(t)|>|\widetilde{c}(t)|+\mu
$$

is valid for $t \geq T_{1}$. It can be easily verified that $\tilde{\gamma}, \tilde{c} \in A C_{\text {loc }}\left(\left[T_{1}, \infty\right), \mathbb{C}\right)$.

For the rest of this section we will denote

$$
\tilde{\vartheta}(t)=\frac{\operatorname{Re}\left(\tilde{\gamma}(t) \tilde{\gamma}^{\prime}(t)-\overline{\widetilde{c}}(t) \tilde{c}^{\prime}(t)\right)-\left|\tilde{\gamma}(t) \tilde{c}^{\prime}(t)-\tilde{\gamma}^{\prime}(t) \tilde{c}(t)\right|}{\tilde{\gamma}^{2}(t)-|\tilde{c}(t)|^{2}} .
$$

The instability and boundedness of solutions are studied subject to suitable subsets of the following assumptions.

(i) The numbers $T_{1} \geq t_{0}, T \geq T_{1}$, and $\mu>0$ are such that (2.3) holds.

(ii) There exist functions $\tilde{\varkappa}_{,} \tilde{\kappa}_{k}, \varrho:[T, \infty) \rightarrow \mathbb{R}$ such that 
Abstract and Applied Analysis

$$
\begin{aligned}
& \left|\tilde{\gamma}(t) g\left(t, z, w_{1}, \ldots, w_{m}\right)+\widetilde{c}(t) \bar{g}\left(t, z, w_{1}, \ldots, w_{m}\right)\right| \\
& \quad \leq \tilde{\varkappa}(t)|\widetilde{\gamma}(t) z+\widetilde{c}(t) \bar{z}|+\sum_{k=1}^{m} \widetilde{\kappa}_{k}(t)\left|\widetilde{\gamma}\left(\theta_{k}(t)\right) w_{k}+\widetilde{c}\left(\theta_{k}(t)\right) \bar{w}_{k}\right|+Q(t)
\end{aligned}
$$

for $t \geq T, z, w_{k} \in \mathbb{C}(k=1, \ldots, m)$, where $\rho$ is continuous on $[T, \infty)$.

$\left(\mathrm{ii}_{\mathrm{n}}\right)$ There exist numbers $R_{n} \geq 0$ and functions $\tilde{\varkappa}_{n}, \tilde{\kappa}_{n k}:[T, \infty) \rightarrow \mathbb{R}$ such that

$$
\begin{aligned}
& \left|\tilde{\gamma}(t) g\left(t, z, w_{1}, \ldots, w_{m}\right)+\widetilde{c}(t) g\left(t, z, w_{1}, \ldots, w_{m}\right)\right| \\
& \quad \leq \tilde{\varkappa}_{n}(t)|\tilde{\gamma}(t) z+\widetilde{c}(t) z|+\sum_{k=1}^{m} \widetilde{\kappa}_{n k}(t)\left|\tilde{\gamma}\left(\theta_{k}(t)\right) w_{k}+\widetilde{c}\left(\theta_{k}(t)\right) \bar{w}_{k}\right|
\end{aligned}
$$

for $t \geq \tau_{n} \geq T,|z|+\sum_{k=1}^{m}\left|w_{k}\right|>R_{n}$.

(iii) $\tilde{\beta} \in A C_{\text {loc }}\left([T, \infty), \mathbb{R}_{-}^{0}\right)$ is a function satisfying

$$
\theta_{k}^{\prime}(t) \tilde{\beta}(t) \leq-\tilde{\lambda}_{k}(t) \quad \text { a.e. on }[T, \infty),
$$

where $\tilde{\lambda}_{k}$ is defined for $t \geq T$ by

$$
\tilde{\mathcal{\lambda}}_{k}(t)=\tilde{\kappa}_{k}(t)+\left(\left|A_{k}(t)\right|+\left|B_{k}(t)\right|\right) \frac{|\widetilde{\gamma}(t)|+|\widetilde{c}(t)|}{\left|\widetilde{\gamma}\left(\theta_{k}(t)\right)\right|-\left|\widetilde{c}\left(\theta_{k}(t)\right)\right|} .
$$

(iii $\left.\left.{ }_{n}\right) \tilde{\beta}_{n} \in A C_{\text {loc }}[T, \infty), \mathbb{R}_{-}^{0}\right)$ is a function satisfying

$$
\theta_{k}^{\prime}(t) \tilde{\beta}_{n}(t) \leq-\tilde{\lambda}_{n k}(t) \quad \text { a.e. on }\left[\tau_{n}, \infty\right)
$$

where $\tilde{\lambda}_{n k}$ is defined for $t \geq T$ by

$$
\tilde{\iota}_{n k}(t)=\widetilde{\kappa}_{n k}(t)+\left(\left|A_{k}(t)\right|+\left|B_{k}(t)\right|\right) \frac{|\widetilde{\gamma}(t)|+|\widetilde{c}(t)|}{\left|\widetilde{\gamma}\left(\theta_{k}(t)\right)\right|-\left|\widetilde{c}\left(\theta_{k}(t)\right)\right|} .
$$

(iv $) \tilde{\Lambda}_{n}$ is a real locally Lebesgue integrable function satisfying the inequalities $\widetilde{\beta}_{n}^{\prime}(t) \geq$ $\tilde{\Lambda}_{n}(t) \tilde{\beta}_{n}(t), \widetilde{\Theta}_{n}(t) \geq \tilde{\Lambda}_{n}(t)$ for almost all $t \in\left[\tau_{n}, \infty\right)$, where $\widetilde{\Theta}_{n}$ is defined by

$$
\tilde{\Theta}_{n}(t)=\operatorname{Re} a(t)+\tilde{\vartheta}(t)-\tilde{\varkappa}_{n}(t)+m \tilde{\beta}_{n}(t)
$$

Obviously, if $A_{k}, B_{k}, \tilde{\kappa}_{k}$, and $\theta_{k}^{\prime}$ are locally absolutely continuous on $[T, \infty)$ and $\tilde{\lambda}_{k}(t) \geq$ $0, \theta_{k}^{\prime}(t)>0$, the choice $\widetilde{\beta}(t)=-\max _{k=1, \ldots, m}\left[\tilde{\lambda}_{k}(t)\left(\theta_{k}^{\prime}(t)\right)^{-1}\right]$ is admissible in (iii). Similarly, if $A_{k}$, 
$B_{k}, \widetilde{\kappa}_{n k}$, and $\theta_{k}^{\prime}$ are locally absolutely continuous on $[T, \infty)$ and $\tilde{\lambda}_{n k}(t) \geq 0, \theta_{k}^{\prime}(t)>0$, the choice $\tilde{\beta}_{n}(t)=-\max _{k=1, \ldots, m}\left[\tilde{\lambda}_{n k}(t)\left(\theta_{k}^{\prime}(t)\right)^{-1}\right]$ is admissible in $\left(\mathrm{iii}_{\mathrm{n}}\right)$.

Denote

$$
\tilde{\Theta}(t)=\operatorname{Re} a(t)+\tilde{\vartheta}(t)-\tilde{\varkappa}(t) .
$$

From assumption (i) it follows that

$$
\begin{aligned}
|\tilde{\vartheta}| & \leq \frac{\left|\operatorname{Re}\left(\tilde{\gamma} \tilde{\gamma}^{\prime}-\overline{\tilde{c}} \tilde{c}^{\prime}\right)\right|+\left|\tilde{\gamma} c^{\prime}-\tilde{\gamma}^{\prime} c\right|}{\tilde{\gamma}^{2}-|\tilde{c}|^{2}} \leq \frac{\left(\left|\tilde{\gamma}^{\prime}\right|+\left|\tilde{c}^{\prime}\right|\right)(|\tilde{\gamma}|+|\widetilde{c}|)}{\tilde{\gamma}^{2}-|\widetilde{c}|^{2}} \\
& =\frac{\left|\tilde{\gamma}^{\prime}\right|+\left|\tilde{c}^{\prime}\right|}{|\tilde{\gamma}|-|\tilde{c}|} \leq \frac{1}{\mu}\left(\left|\tilde{\gamma}^{\prime}\right|+\left|\tilde{c}^{\prime}\right|\right) ;
\end{aligned}
$$

therefore the function $\tilde{\vartheta}$ is locally Lebesgue integrable on $[T, \infty)$, assuming that (i) holds true. If the relations $\tilde{\beta}_{n} \in A C_{\mathrm{loc}}\left([T, \infty), \mathbb{R}_{-}\right), \tilde{\varkappa}_{n} \in L_{\mathrm{loc}}([T, \infty), \mathbb{R})$, and $\tilde{\beta}_{n}^{\prime}(t) / \tilde{\beta}_{n}(t) \leq \widetilde{\Theta}_{n}(t)$ for almost all $t \geq \tau_{n}$ together with conditions (i) and (iii $)$ are fulfilled, then we can choose $\widetilde{\Lambda}_{n}(t)=\widetilde{\Theta}_{n}(t)$ for $t \in[T, \infty)$ in $\left(\mathrm{iv}_{\mathrm{n}}\right)$.

\section{Results}

Theorem 3.1. Let assumptions ( $i$ ), $\left(i i_{0}\right)$, ( $\left.i i_{0}\right)$, and $\left(i v_{0}\right)$ be fulfilled for some $\tau_{0} \geq T$. Suppose there exist $t_{1} \geq \tau_{0}$ and $v \in(-\infty, \infty)$ such that

$$
\inf _{t \geq t_{1}}\left[\int_{t_{1}}^{t} \tilde{\Lambda}_{0}(s) d s-\ln (|\tilde{\gamma}(t)|+|\widetilde{c}(t)|)\right] \geq v
$$

If $z(t)$ is any solution of (1.2) satisfying

$$
\min _{\theta\left(t_{1}\right) \leq s \leq t_{1}}|z(s)|>R_{0}, \quad \Delta\left(t_{1}\right)>R_{0} e^{-v}
$$

where

$$
\begin{gathered}
\theta(t)=\min _{k=1, \ldots, m} \theta_{k}(t), \\
\Delta(t)=(|\widetilde{\gamma}(t)|-|\widetilde{c}(t)|)|z(t)|+\tilde{\beta}_{0}(t) \max _{\theta(t) \leq s \leq t}|z(s)| \sum_{k=1}^{m} \int_{\theta_{k}(t)}^{t}(|\widetilde{\gamma}(s)|+|\widetilde{c}(s)|) d s,
\end{gathered}
$$


then

$$
|z(t)| \geq \frac{\Delta\left(t_{1}\right)}{|\tilde{\gamma}(t)|+|\tilde{c}(t)|} \exp \left[\int_{t_{1}}^{t} \tilde{\Lambda}_{0}(s) d s\right]
$$

for all $t \geq t_{1}$, for which $z(t)$ is defined.

In the proof we use the following Lemma.

Lemma 3.2. Let $a_{1}, a_{2}, b_{1}, b_{2} \in \mathbb{C},\left|a_{2}\right|>\left|b_{2}\right|$. Then,

$$
\operatorname{Re} \frac{a_{1} z+b_{1} \bar{z}}{a_{2} z+b_{2} \bar{z}} \geq \frac{\operatorname{Re}\left(a_{1} \bar{a}_{2}-b_{1} \bar{b}_{2}\right)-\left|a_{1} b_{2}-a_{2} b_{1}\right|}{\left|a_{2}\right|^{2}-\left|b_{2}\right|^{2}}
$$

for $z \in \mathbb{C}, z \neq 0$.

The proof is analogous to that of Lemma 1 in [20, page 101] or to the proof of Lemma in [16, page 131].

Proof of Theorem 3.1. Let $z(t)$ be any solution of (1.2) satisfying (3.2). Consider the Lyapunov functional

$$
V(t)=U(t)+\tilde{\beta}_{0}(t) \sum_{k=1}^{m} \int_{\theta_{k}(t)}^{t} U(s) d s,
$$

where

$$
U(t)=|\tilde{\gamma}(t) z(t)+\widetilde{c}(t) \bar{z}(t)|
$$

For brevity we shall denote $w_{k}(t)=z\left(\theta_{k}(t)\right)$ and we shall write the function of variable $t$ simply without indicating the variable $t$, for example, $\tilde{\gamma}$ instead of $\tilde{\gamma}(t)$.

In view of (3.6), we have

$$
\begin{aligned}
V^{\prime}= & U^{\prime}+\tilde{\beta}_{0}^{\prime} \sum_{k=1}^{m} \int_{\theta_{k}(t)}^{t} U(s) d s+m \tilde{\beta}_{0}|\tilde{\gamma} z+\widetilde{c} z| \\
& -\sum_{k=1}^{m} \theta_{k}^{\prime} \tilde{\beta}_{0}\left|\tilde{\gamma}\left(\theta_{k}(t)\right) w_{k}+\widetilde{c}\left(\theta_{k}(t)\right) \bar{w}_{k}\right|
\end{aligned}
$$

for almost all $t \geq t_{1}$ for which $z(t)$ is defined and $U^{\prime}(t)$ exists. Put $\mathcal{K}=\left\{t \geq t_{1}\right.$ : $z(t)$ exists, $\left.|z(t)|>R_{0}\right\}$. Clearly $U(t) \neq 0$ for $t \in \mathcal{K}$. The derivative $U^{\prime}(t)$ exists for almost all $t \in \mathcal{K}$. 
Since $z(t)$ is a solution of (1.2), we obtain

$$
\begin{aligned}
& U U^{\prime}=\operatorname{Re}\left[(\tilde{\gamma} \bar{z}+\overline{\widetilde{c}} z)\left(\tilde{\gamma}^{\prime} z+\tilde{\gamma} z^{\prime}+\tilde{c}^{\prime} \bar{z}+\tilde{c} \bar{z}^{\prime}\right)\right] \\
& =\operatorname{Re}\left\{( \tilde { \gamma } \overline { z } + \overline { \tilde { c } } z ) \left[\tilde{\gamma}^{\prime} z+\tilde{c}^{\prime} \bar{z}+\tilde{\gamma}\left(a z+b \bar{z}+\sum_{k=1}^{m}\left(A_{k} w_{k}+B_{k} \bar{w}_{k}\right)+g\right)\right.\right. \\
& \left.\left.+\tilde{c}(\overline{a z}+\bar{b} z)+\sum_{k=1}^{m}\left(\bar{A}_{k} \bar{w}_{k}+\bar{B}_{k} w_{k}+\bar{g}\right)\right]\right\} \\
& =\operatorname{Re}\left\{( \tilde { \gamma } \overline { z } + \overline { \tilde { c } } z ) \left[\tilde{\gamma}^{\prime} z+\tilde{c}^{\prime} \bar{z}+(\tilde{\gamma} a+\tilde{c} \bar{b}) z+(\tilde{\gamma} b+\widetilde{c} \bar{a}) \bar{z}\right.\right. \\
& \left.\left.+\tilde{\gamma}\left(\sum_{k=1}^{m}\left(A_{k} w_{k}+B_{k} \bar{w}_{k}\right)+g\right)+\widetilde{c}\left(\sum_{k=1}^{m}\left(\bar{A}_{k} \bar{w}_{k}+\bar{B}_{k} w_{k}\right)+\bar{g}\right)\right]\right\}
\end{aligned}
$$

for almost all $t \in \mathcal{K}$. Taking into account

$$
(\tilde{\gamma} a+\tilde{c} \bar{b}) \tilde{c}=(\tilde{\gamma} b+\widetilde{c} a) \tilde{\gamma}
$$

we get

$$
\begin{aligned}
& U U^{\prime} \geq \operatorname{Re}\left\{(\tilde{\gamma} \bar{z}+\overline{\tilde{c}} z)(\tilde{\gamma} a+\tilde{c} \bar{b})\left(z+\frac{\tilde{c}}{\tilde{\gamma}} \bar{z}\right)\right\} \\
& +\operatorname{Re}\left\{(\tilde{r} \bar{z}+\overline{\tilde{c}} z)\left[\tilde{r} \sum_{k=1}^{m}\left(A_{k} w_{k}+B_{k} \bar{w}_{k}\right)+\tilde{c} \sum_{k=1}^{m}\left(\bar{A}_{k} \bar{w}_{k}+\bar{B}_{k} w_{k}\right)\right]\right\} \\
& +\operatorname{Re}\{(\tilde{\gamma} \bar{z}+\overline{\tilde{c}} z)(\tilde{\gamma} g+\widetilde{c} \bar{g})\}+\operatorname{Re}\left\{(\tilde{\gamma} \bar{z}+\overline{\tilde{c}} z)\left(\tilde{\gamma}^{\prime} z+\tilde{c}^{\prime} \bar{z}\right)\right\} \\
& \geq U^{2} \operatorname{Re}\left(a+\frac{\tilde{c}}{\tilde{r}} \bar{b}\right)-U(|\tilde{r}|+|\tilde{c}|)\left(\sum_{k=1}^{m}\left|A_{k} w_{k}+B_{k} \bar{w}_{k}\right|\right) \\
& -U|\tilde{\gamma} g+\widetilde{c} \bar{g}|+U^{2} \operatorname{Re} \frac{\tilde{\gamma}^{\prime} z+\tilde{c}^{\prime} \bar{z}}{\tilde{\gamma} z+\tilde{c} \bar{z}} .
\end{aligned}
$$

By the use of Lemma 3.2, we get

$$
\operatorname{Re} \frac{\tilde{\gamma}^{\prime} z+\tilde{c}^{\prime} \bar{z}}{\tilde{\gamma} z+\tilde{c} z} \geq \tilde{\vartheta}
$$
relation

The last inequality together with (2.12), taken for $n=0$, assumption ( $\left.\mathrm{ii}_{0}\right)$, and the

$$
\operatorname{Re}\left(a+\frac{\tilde{c}}{\tilde{\gamma}} \bar{b}\right)=\operatorname{Re} a
$$


yields

$$
\begin{aligned}
& U U^{\prime} \geq U^{2}\left(\operatorname{Re} a+\tilde{\vartheta}-\tilde{\varkappa}_{0}\right)-U \sum_{k=1}^{m}\left(\tilde{\kappa}_{0 k}\left|\tilde{\gamma}\left(\theta_{k}\right) w_{k}+\tilde{c}\left(\theta_{k}\right) \bar{w}_{k}\right|\right) \\
& -U(|\tilde{\gamma}|+|\widetilde{c}|)\left(\sum_{k=1}^{m} \frac{\left|A_{k}\right|\left|w_{k}\right|+\left|B_{k}\right|\left|\bar{w}_{k}\right|}{\left|\tilde{\gamma}\left(\theta_{k}\right)\right|-\left|\widetilde{c}\left(\theta_{k}\right)\right|}\left(\left|\tilde{\gamma}\left(\theta_{k}\right)\right|-\left|\widetilde{c}\left(\theta_{k}\right)\right|\right)\right) \\
& \geq U^{2}\left(\operatorname{Re} a+\tilde{\vartheta}-\tilde{\varkappa}_{0}\right) \\
& -U\left\{\sum_{k=1}^{m}\left[\tilde{\kappa}_{0 k}+\left(\left|A_{k}\right|+\left|B_{k}\right|\right) \frac{|\tilde{\gamma}|+|\widetilde{c}|}{\left|\widetilde{\gamma}\left(\theta_{k}\right)\right|-\left|\widetilde{c}\left(\theta_{k}\right)\right|}\right]\left|\widetilde{\gamma}\left(\theta_{k}\right) w_{k}+\widetilde{c}\left(\theta_{k}\right) \bar{w}_{k}\right|\right\} \\
& \geq U^{2}\left(\operatorname{Re} a+\tilde{\vartheta}-\tilde{\varkappa}_{0}\right)-U \sum_{k=1}^{m} \tilde{\lambda}_{0 k}\left|\tilde{r}\left(\theta_{k}\right) w_{k}+\tilde{c}\left(\theta_{k}\right) \bar{w}_{k}\right|
\end{aligned}
$$

for almost all $t \in \mathcal{K}$.

Consequently,

$$
U^{\prime} \geq U\left(\operatorname{Re} a+\tilde{\vartheta}-\tilde{\varkappa}_{0}\right)-\sum_{k=1}^{m} \tilde{\lambda}_{0 k}\left|\tilde{\gamma}\left(\theta_{k}\right) w_{k}+\tilde{c}\left(\theta_{k}\right) \bar{w}_{k}\right|
$$

for almost all $t \in \mathcal{K}$. Inequality (3.15) together with relation (3.8) gives

$$
\begin{aligned}
V^{\prime} \geq & U\left(\operatorname{Re} a+\tilde{\vartheta}-\tilde{\varkappa}_{0}+m \tilde{\beta}_{0}\right)-\sum_{k=1}^{m}\left(\tilde{\lambda}_{0 k}+\theta_{k}^{\prime} \tilde{\beta}_{0}\right)\left|\tilde{\gamma}\left(\theta_{k}\right) w_{k}+\tilde{c}\left(\theta_{k}\right) \bar{w}_{k}\right| \\
& +\tilde{\beta}_{0}^{\prime} \sum_{k=1}^{m} \int_{\theta_{k}(t)}^{t}|\tilde{\gamma}(s) z(s)+\tilde{c}(s) \bar{z}(s)| d s .
\end{aligned}
$$

Using (2.11) and (2.13) for $n=0$, we obtain

$$
V^{\prime}(t) \geq U(t) \widetilde{\Theta}_{0}(t)+\tilde{\beta}_{0}^{\prime}(t) \sum_{k=1}^{m} \int_{\theta_{k}(t)}^{t} U(s) d s
$$

Hence, in view of $\left(\mathrm{iv}_{0}\right)$,

$$
V^{\prime}(t)-\tilde{\Lambda}_{0}(t) V(t) \geq 0
$$

for almost all $t \in \mathcal{K}$.

Multiplying (3.18) by $\exp \left[-\int_{t_{1}}^{t} \tilde{\Lambda}_{0}(s) d s\right]$ and integrating over $\left[t_{1}, t\right]$, we get

$$
V(t) \exp \left[-\int_{t_{1}}^{t} \tilde{\Lambda}_{0}(s) d s\right]-V\left(t_{1}\right) \geq 0
$$


on any interval $\left[t_{1}, \omega\right)$, where the solution $z(t)$ exists and satisfies the inequality $|z(t)|>R_{0}$. Now, with respect to (3.6), (3.7), and $\tilde{\beta}_{0} \leq 0$, we have

$$
(|\tilde{\gamma}(t)|+|\widetilde{c}(t)|)|z(t)| \geq V(t) \geq V\left(t_{1}\right) \exp \left[\int_{t_{1}}^{t} \tilde{\Lambda}_{0}(s) d s\right] \geq \Delta\left(t_{1}\right) \exp \left[\int_{t_{1}}^{t} \tilde{\Lambda}_{0}(s) d s\right]
$$

If (3.2) is fulfilled, there is $R>R_{0}$ such that $\Delta\left(t_{1}\right)>R e^{-v}$. By virtue of (3.1), and (3.2), we can easily see that

$$
|z(t)| \geq \frac{\Delta\left(t_{1}\right)}{|\widetilde{\gamma}(t)|+|\tilde{c}(t)|} \exp \left[\int_{t_{1}}^{t} \tilde{\Lambda}_{0}(s) d s\right] \geq R e^{-v} e^{v}=R
$$

for all $t \geq t_{1}$, for which $z(t)$ is defined.

To obtain results on the existence of bounded solutions, we shall suppose that (1.2) satisfies the uniqueness property of solutions. Moreover, we suppose that the delays are bounded, that is, that the functions $\theta_{k}$ satisfy the condition

$$
t-r \leq \theta_{k}(t) \leq t \quad \text { for } t \geq t_{0}+r,
$$

where $r>0$ is a constant. Our assumptions imply the existence of numbers $T_{1}=t_{0}+r, T \geq T_{1}$, and $\mu>0$ such that

$$
|\operatorname{Im} a(t)|>|b(t)|+\mu \quad \text { for } t \geq T_{1}, \quad t \geq \theta_{k}(t) \geq t-r \quad \text { for } t \geq T(k=1, \ldots, m) .
$$

In view of this, we replace (2.3) in assumption (i) with $\left(5^{\prime}\right)$. All other assumptions we keep in validity.

In the proof of the following theorem we shall utilize Ważewski topological principle for retarded functional differential equations of Carathéodory type. Details of this theory can be found in the paper of Rybakowski [15].

Theorem 3.3. Let conditions (i), (ii), and (iii) be fulfilled, and let $\tilde{\Lambda}, \theta_{k}^{\prime} \quad(k=1, \ldots, m)$ be continuous functions such that the inequality $\tilde{\Lambda}(t) \leq \widetilde{\Theta}(t)$ holds a.e. on $[T, \infty)$, where $\widetilde{\Theta}$ is defined by (2.14). Suppose that $\xi:[T-r, \infty) \rightarrow \mathbb{R}$ is a continuous function such that

$$
\tilde{\Lambda}(t)+\tilde{\beta}(t) \sum_{k=1}^{m} \theta_{k}^{\prime}(t) \exp \left[-\int_{\theta_{k}(t)}^{t} \xi(s) d s\right]-\xi(t)>\varphi(t) C^{-1} \exp \left[-\int_{T}^{t} \xi(s) d s\right]
$$


for $t \in[T, \infty]$ and some constant $C>0$. Then, there exist $t_{2}>T$ and a solution $z_{0}(t)$ of $(1.2)$ satisfying

$$
\left|z_{0}(t)\right| \leq \frac{C}{|\widetilde{\gamma}(t)|-|\widetilde{c}(t)|} \exp \left[\int_{T}^{t} \xi(s) d s\right]
$$

for $t \geq t_{2}$.

Proof. Write (1.2) in the form

$$
z^{\prime}=F\left(t, z_{t}\right),
$$

where $F: J \times \mathcal{C} \rightarrow \mathbb{C}$ is defined by

$$
\begin{aligned}
F(t, \psi)= & a(t) \psi(0)+b(t) \bar{\psi}(0)+\sum_{k=1}^{m}\left[A_{k}(t) \psi\left(\theta_{k}(t)-t\right)+B_{k}(t) \bar{\psi}\left(\theta_{k}(t)-t\right)\right] \\
& +g\left(t, \psi(0), \psi\left(\theta_{1}(t)-t\right), \ldots, \psi\left(\theta_{m}(t)-t\right)\right)
\end{aligned}
$$

and $z_{t}$ is the element of $\mathcal{C}$ defined by the relation $z_{t}(\tilde{\theta})=z(t+\tilde{\theta}), \tilde{\theta} \in[-r, 0]$. Let $\tau>T$. Put

$$
\begin{aligned}
\tilde{U}(t, z, \bar{z}) & =|\tilde{r}(t) z+\tilde{c}(t) \bar{z}|-\varphi(t), \\
\varphi(t) & =C \exp \left[\int_{T}^{t} \xi(s) d s\right], \\
\Omega^{0} & =\{(t, z) \in(\tau, \infty) \times \mathbb{C}: \tilde{U}(t, z, \bar{z})<0\}, \\
\Omega_{\tilde{U}} & =\{(t, z) \in(\tau, \infty) \times \mathbb{C}: \tilde{U}(t, z, \bar{z})=0\} .
\end{aligned}
$$

It can be easily verified that $\Omega^{0}$ is a polyfacial set generated by the functions $\widehat{U}(t)=\tau-t$, $\tilde{U}(t, z, \bar{z})$ (see Rybakowski [15, page 134]). It holds that $\Omega_{\tilde{U}} \subset \partial \Omega^{0}$. As $(|\widetilde{\gamma}(t)|+|\widetilde{c}(t)|)|z(t)| \geq$ $|\tilde{\gamma}(t) z+\widetilde{c}(t) \bar{z}|$, we have

$$
|z| \geq \frac{\varphi(t)}{|\tilde{r}(t)|+|\tilde{c}(t)|}=\frac{C}{|\tilde{r}(t)|+|\widetilde{c}(t)|} \exp \left[\int_{T}^{t} \xi(s) d s\right]>0
$$

for $(t, z) \in \Omega_{\tilde{U}}$. It holds that

$$
D^{+} \widehat{U}(t)=\frac{\partial}{\partial t}(\tau-t)=-1<0
$$


Let $\left(t^{*}, \zeta\right) \in \Omega_{\tilde{U}}$ and $\phi \in \mathcal{C}$ be such that $\phi(0)=\zeta$ and $\left(t^{*}+\tilde{\theta}, \phi(\tilde{\theta})\right) \in \Omega^{0}$ for all $\tilde{\theta} \in[-r, 0)$. If $(t, \psi) \in(\tau, \infty) \times \mathcal{C}$, then

$$
\begin{aligned}
D^{+} \tilde{U}(t, \psi(0), \bar{\psi}(0)):= & \limsup _{h \rightarrow 0+}\left(\frac{1}{h}\right)[\tilde{U}(t+h, \psi(0)+h F(t, \psi), \bar{\psi}(0)+h \bar{F}(t, \psi)) \\
-\tilde{U}(t, \psi(0), \bar{\psi}(0))] & \\
= & \frac{\partial \tilde{U}(t, \psi(0), \bar{\psi}(0))}{\partial t}+\frac{\partial \tilde{U}(t, \psi(0), \bar{\psi}(0))}{\partial z} F(t, \psi) \\
& +\frac{\partial \tilde{U}(t, \psi(0), \bar{\psi}(0))}{\partial \bar{z}} \bar{F}(t, \psi) .
\end{aligned}
$$

Therefore,

$$
\begin{aligned}
D^{+} \tilde{U}(t, \psi(0), \bar{\psi}(0))= & |\widetilde{\gamma}(t) \psi(0)+\widetilde{c}(t) \bar{\psi}(0)| \operatorname{Re} \frac{\tilde{\gamma}^{\prime}(t) \psi(0)+\tilde{c}^{\prime}(t) \bar{\psi}(0)}{\widetilde{\gamma}(t) \psi(0)+\widetilde{c}(t) \bar{\psi}(0)}-\varphi^{\prime}(t) \\
& +\frac{1}{2}|\widetilde{\gamma}(t) \psi(0)+\widetilde{c}(t) \bar{\psi}(0)|^{-1} \\
\times & \{[\widetilde{\gamma}(t)(\widetilde{\gamma}(t) \bar{\psi}(0)+\overline{\widetilde{c}}(t) \psi(0))+(\widetilde{\gamma}(t) \psi(0)+\widetilde{c}(t) \bar{\psi}(0)) \overline{\widetilde{c}}(t)] F(t, \psi) \\
& +[\widetilde{c}(t)(\tilde{\gamma}(t) \bar{\psi}(0)+\overline{\widetilde{c}}(t) \psi(0))+\widetilde{\gamma}(t)(\tilde{\gamma}(t) \psi(0)+\widetilde{c}(t) \bar{\psi}(0))] \bar{F}(t, \psi)\}
\end{aligned}
$$

provided that the derivatives $\tilde{\gamma}^{\prime}(t), \tilde{c}^{\prime}(t)$ exist and that $\psi(0) \neq 0$. Thus,

$$
\begin{aligned}
& D^{+} \tilde{U}(t, \psi(0), \bar{\psi}(0))=|\tilde{\gamma}(t) \psi(0)+\widetilde{c}(t) \bar{\psi}(0)| \operatorname{Re} \frac{\tilde{\gamma}^{\prime}(t) \psi(0)+\tilde{c^{\prime}}(t) \bar{\psi}(0)}{\tilde{\gamma}(t) \psi(0)+\tilde{c}(t) \bar{\psi}(0)}-\varphi^{\prime}(t) \\
& +|\tilde{\gamma}(t) \psi(0)+\tilde{c}(t) \bar{\psi}(0)|^{-1} \operatorname{Re}\{\tilde{\gamma}(t)(\tilde{\gamma}(t) \bar{\psi}(0)+\overline{\widetilde{c}}(t) \psi(0)) F(t, \psi) \\
& +\widetilde{c}(t)(\tilde{\gamma}(t) \bar{\psi}(0)+\overline{\widetilde{c}}(t) \psi(0)) \bar{F}(t, \psi)\} \\
& =|\tilde{\gamma}(t) \psi(0)+\tilde{c}(t) \bar{\psi}(0)| \operatorname{Re} \frac{\tilde{\gamma}^{\prime}(t) \psi(0)+\tilde{c^{\prime}}(t) \bar{\psi}(0)}{\tilde{\gamma}(t) \psi(0)+\widetilde{c}(t) \bar{\psi}(0)}-\varphi^{\prime}(t) \\
& +|\widetilde{\gamma}(t) \psi(0)+\widetilde{c}(t) \bar{\psi}(0)|^{-1} \operatorname{Re}\{(\tilde{\gamma}(t) \bar{\psi}(0)+\overline{\widetilde{c}}(t) \psi(0)) \\
& \times(\tilde{\gamma}(t) F(t, \psi)+\tilde{c}(t) \bar{F}(t, \psi))\} .
\end{aligned}
$$

Using (3.10), (3.13), and (ii), similarly to the proof of Theorem 3.1, we obtain

$$
\begin{aligned}
D^{+} \tilde{U}(t, \psi(0), \bar{\psi}(0)) \geq & |\tilde{\gamma}(t) \psi(0)+\widetilde{c}(t) \bar{\psi}(0)| \operatorname{Re} a(t) \\
& -\sum_{k=1}^{m}\left|A_{k}(t) \psi\left(\theta_{k}(t)-t\right)+B_{k}(t) \bar{\psi}\left(\theta_{k}(t)-t\right)\right|(|\widetilde{\gamma}(t)|+|\widetilde{c}(t)|) \\
& -\tilde{\varkappa}(t)|\widetilde{\gamma}(t) \psi(0)+\widetilde{c}(t) \bar{\psi}(0)|
\end{aligned}
$$


Abstract and Applied Analysis

$$
\begin{aligned}
& -\sum_{k=1}^{m} \widetilde{\kappa}_{k}(t)\left|\widetilde{\gamma}\left(\theta_{k}(t)\right) \psi\left(\theta_{k}(t)-t\right)+\widetilde{c}\left(\theta_{k}(t)\right) \bar{\psi}\left(\theta_{k}(t)-t\right)\right| \\
& +\widetilde{\vartheta}(t)|\widetilde{\gamma}(t) \psi(0)+\widetilde{c}(t) \bar{\psi}(0)|-\rho(t)-\varphi^{\prime}(t)
\end{aligned}
$$

and consequently, with respect to (iii),

$$
\begin{aligned}
& D^{+} \tilde{U}(t, \psi(0), \bar{\psi}(0)) \geq(\operatorname{Re} a(t)+\tilde{\vartheta}(t)-\tilde{\varkappa}(t))|\tilde{\gamma}(t) \psi(0)+\widetilde{c}(t) \bar{\psi}(0)| \\
& -\sum_{k=1}^{m} \tilde{\lambda}_{k}(t)\left|\widetilde{\gamma}\left(\theta_{k}(t)\right) \psi\left(\theta_{k}(t)-t\right)+\widetilde{c}\left(\theta_{k}(t)\right) \bar{\psi}\left(\theta_{k}(t)-t\right)\right|-\rho(t)-\varphi^{\prime}(t) \\
& \geq \widetilde{\Theta}(t)|\widetilde{\gamma}(t) \psi(0)+\widetilde{c}(t) \bar{\psi}(0)| \\
& +\widetilde{\beta}(t) \sum_{k=1}^{m} \theta_{k}^{\prime}(t)\left|\widetilde{\gamma}\left(\theta_{k}(t)\right) \psi\left(\theta_{k}(t)-t\right)+\widetilde{c}\left(\theta_{k}(t)\right) \bar{\psi}\left(\theta_{k}(t)-t\right)\right|-\rho(t)-\varphi^{\prime}(t) \\
& \geq \widetilde{\Lambda}(t)|\widetilde{\gamma}(t) \psi(0)+\widetilde{c}(t) \bar{\psi}(0)| \\
& +\tilde{\beta}(t) \sum_{k=1}^{m} \theta_{k}^{\prime}(t)\left|\tilde{\gamma}\left(\theta_{k}(t)\right) \psi\left(\theta_{k}(t)-t\right)+\tilde{c}\left(\theta_{k}(t)\right) \bar{\psi}\left(\theta_{k}(t)-t\right)\right|-\rho(t)-\varphi^{\prime}(t)
\end{aligned}
$$

for almost all $t \in(\tau, \infty)$ and for $\psi \in \mathcal{C}$ sufficiently close to $\phi$. Replacing $t$ and $\psi$ by $t^{*}$ and $\phi$, respectively, in the last expression, we get

$$
\begin{aligned}
& \tilde{\Lambda}\left(t^{*}\right)\left|\tilde{\gamma}\left(t^{*}\right) \phi(0)+\tilde{c}\left(t^{*}\right) \bar{\phi}(0)\right|+\tilde{\beta}\left(t^{*}\right) \sum_{k=1}^{m} \theta_{k}^{\prime}\left(t^{*}\right)\left|\tilde{r}\left(\theta_{k}\left(t^{*}\right)\right) \phi\left(\theta_{k}\left(t^{*}\right)-t^{*}\right)+\tilde{c}\left(\theta_{k}\left(t^{*}\right)\right) \bar{\phi}\left(\theta_{k}\left(t^{*}\right)-t^{*}\right)\right| \\
& -\rho\left(t^{*}\right)-\varphi^{\prime}\left(t^{*}\right) \\
& \geq \widetilde{\Lambda}\left(t^{*}\right)\left|\tilde{\gamma}\left(t^{*}\right) \zeta+\widetilde{c}\left(t^{*}\right) \bar{\zeta}\right|+\tilde{\beta}\left(t^{*}\right) \sum_{k=1}^{m} \theta_{k}^{\prime}\left(t^{*}\right) \varphi\left(\theta_{k}\left(t^{*}\right)\right)-\rho\left(t^{*}\right)-\varphi^{\prime}\left(t^{*}\right) \\
& \geq \tilde{\Lambda}\left(t^{*}\right) \varphi\left(t^{*}\right)+\tilde{\beta}\left(t^{*}\right) \sum_{k=1}^{m} \theta_{k}^{\prime}\left(t^{*}\right) \varphi\left(\theta_{k}\left(t^{*}\right)\right)-\varphi\left(t^{*}\right)-\varphi^{\prime}\left(t^{*}\right) \\
& =\tilde{\Lambda}\left(t^{*}\right) C \exp \left[\int_{T}^{t^{*}} \xi(s) d s\right]+\tilde{\beta}\left(t^{*}\right) \sum_{k=1}^{m} \theta_{k}^{\prime}\left(t^{*}\right) C \exp \left[\int_{T}^{\theta_{k}\left(t^{*}\right)} \xi(s) d s\right] \\
& -\rho\left(t^{*}\right)-C \xi\left(t^{*}\right) \exp \left[\int_{T}^{t^{*}} \xi(s) d s\right] \\
& =\left\{\tilde{\Lambda}\left(t^{*}\right)+\tilde{\beta}\left(t^{*}\right) \sum_{k=1}^{m} \theta_{k}^{\prime}\left(t^{*}\right) \exp \left[-\int_{\theta_{k}\left(t^{*}\right)}^{t^{*}} \xi(s) d s\right]-\xi\left(t^{*}\right)\right\} C \exp \left[\int_{T}^{t^{*}} \xi(s) d s\right] \\
& -\rho\left(t^{*}\right)>0 \text {. }
\end{aligned}
$$


Therefore, in view of the continuity, $D^{+} \tilde{U}(t, \psi(0), \bar{\psi}(0))>0$ holds for $\psi$ sufficiently close to $\phi$ and almost all $t$ sufficiently close to $t^{*}$. Hence, $\Omega^{0}$ is a regular polyfacial set with respect to $\left(2^{\prime}\right)$.

Choose $Z=\left\{\left(t_{2}, z\right) \in \Omega^{0} \cup \Omega_{\tilde{U}}\right\}$, where $t_{2}>\tau+r$ is fixed. It can be easily verified that $Z \cap \Omega_{\tilde{U}}$ is a retract of $\Omega_{\tilde{U}}$, but $Z \cap \Omega_{\tilde{U}}$ is not a retract of $Z$. Let $\eta \in \mathcal{C}$ be such that $\eta(0)=1$ and $0 \leq \eta(\theta)<1$ for $\theta \in[-r, 0)$. Define the mapping $p: Z \rightarrow \mathcal{C}$ for $\left(t_{2}, z\right) \in Z$ by the relation

$$
\begin{aligned}
p\left(t_{2}, z\right)(\theta)=\frac{\varphi\left(t_{2}+\theta\right) \eta(\theta)}{\left(\tilde{\gamma}^{2}\left(t_{2}+\theta\right)-\left|\widetilde{c}\left(t_{2}+\theta\right)\right|^{2}\right) \varphi\left(t_{2}\right)}\left[\left(\tilde{\gamma}\left(t_{2}\right) \tilde{\gamma}\left(t_{2}+\theta\right)-\overline{\tilde{c}}\left(t_{2}\right) \tilde{c}\left(t_{2}+\theta\right)\right) z\right. \\
\left.+\left(\tilde{\gamma}\left(t_{2}+\theta\right) \tilde{c}\left(t_{2}\right)-\tilde{\gamma}\left(t_{2}\right) \tilde{c}\left(t_{2}+\theta\right)\right) \bar{z}\right]
\end{aligned}
$$

The mapping $p$ is continuous, and it holds that

$$
p\left(t_{2}, z\right)(0)=z \quad \text { for }\left(t_{2}, z\right) \in Z, \quad p\left(t_{2}, 0\right)(\theta)=0 \quad \text { for } \theta \in[-r, 0] .
$$

Since

$$
\tilde{\gamma}\left(t_{2}+\theta\right) p\left(t_{2}, z\right)(\theta)+\widetilde{c}\left(t_{2}+\theta\right) \overline{p\left(t_{2}, z\right)(\theta)}=\frac{\varphi\left(t_{2}+\theta\right) \eta(\theta)}{\varphi\left(t_{2}\right)}\left(\tilde{\gamma}\left(t_{2}\right) z+\tilde{c}\left(t_{2}\right) \bar{z}\right),
$$

we have

$$
\begin{gathered}
\left|\tilde{\gamma}\left(t_{2}\right) z+\tilde{c}\left(t_{2}\right) \bar{z}\right|<\varphi\left(t_{2}\right) \\
\left|\tilde{\gamma}\left(t_{2}+\theta\right) p\left(t_{2}, z\right)(\theta)+\widetilde{c}\left(t_{2}+\theta\right) \overline{p\left(t_{2}, z\right)(\theta)}\right|<\varphi\left(t_{2}+\theta\right)
\end{gathered}
$$

for $\left(t_{2}, z\right) \in Z \cap \Omega^{0}$ and $\theta \in[-r, 0]$. Clearly, inequality (3.39) holds also for $\left(t_{2}, z\right) \in Z \cap \Omega_{\tilde{u}}$ and $\theta \in[-r, 0)$.

Using a topological principle for retarded functional differential equations (see Rybakowski [15, Theorem 2.1]), we see that there is a solution $z_{0}(t)$ of (1.2) such that $\left(t, z_{0}(t)\right) \in \Omega^{0}$ for all $t \geq t_{2}$ for which the solution $z_{0}(t)$ exists. Obviously, $z_{0}(t)$ exists for all $t \geq t_{2}$ and

$$
(|\tilde{\gamma}(t)|-|\tilde{c}(t)|)\left|z_{0}(t)\right| \leq\left|\tilde{\gamma}(t) z_{0}(t)+\widetilde{c}(t) \bar{z}_{0}(t)\right| \leq \varphi(t) \quad \text { for } t \geq t_{2}
$$

Hence

$$
\left|z_{0}(t)\right| \leq \frac{\varphi(t)}{|\widetilde{\gamma}(t)|-|\widetilde{c}(t)|} \quad \text { for } t \geq t_{2}
$$

Theorem 3.4. Suppose that hypotheses (i), $(\underset{\sim}{\sim}),\left(i i_{n}\right),(i i i),\left(i i_{n}\right)$, and (iv $\left.{ }_{n}\right)$ are fulfilled for $\tau_{n} \geq T$ and $n \in \mathbb{N}$, where $R_{n}>0, \inf _{n \in \mathbb{N}} R_{n}=0$. Let $\widetilde{\Lambda}, \theta_{k}^{\prime}$ be continuous functions satisfying the inequality 
$\tilde{\Lambda}(t) \leq \widetilde{\Theta}(t)$ a.e. on $[T, \infty)$, where $\widetilde{\Theta}$ is defined by (2.14). Assume that $\xi:[T-r, \infty) \rightarrow \mathbb{R}$ is a continuous function such that

$$
\tilde{\Lambda}(t)+\tilde{\beta}(t) \sum_{k=1}^{m} \theta_{k}^{\prime}(t) \exp \left[-\int_{\theta_{k}(t)}^{t} \xi(s) d s\right]-\xi(t)>\rho(t) C^{-1} \exp \left(-\int_{T}^{t} \xi(s) d s\right)
$$

for $t \in[T, \infty)$ and some constant $C>0$. Suppose that

$$
\begin{gathered}
\limsup _{t \rightarrow \infty}\left[\int_{T}^{t}\left(\tilde{\Lambda}_{n}(s)-\xi(s)\right) d s+\ln \frac{|\tilde{\gamma}(t)|-|\widetilde{c}(t)|}{|\tilde{\gamma}(t)|+|\widetilde{c}(t)|}\right]=\infty, \\
\lim _{t \rightarrow \infty}\left[\tilde{\beta}_{n}(t) \max _{\theta(t) \leq s \leq t} \frac{\exp \left[\int_{T}^{s} \xi(\sigma) d \sigma\right]}{|\tilde{\gamma}(s)|-|\tilde{c}(s)|} \sum_{k=1}^{m} \int_{\theta_{k}(t)}^{t}(|\widetilde{\gamma}(s)|+|\widetilde{c}(s)|) d s\right]=0, \\
\inf _{\tau_{n} \leq s \leq t<\infty}\left[\int_{s}^{t} \tilde{\Lambda}_{n}(\sigma) d \sigma-\ln (|\tilde{\gamma}(t)|+|\widetilde{c}(t)|)\right] \geq \mathcal{v}
\end{gathered}
$$

for $n \in \mathbb{N}$, where $\theta(t)=\min _{k=1, \ldots, m} \theta_{k}(t)$ and $v \in(-\infty, \infty)$. Then, there exists a solution $z_{0}(t)$ of (1.2) such that

$$
\lim _{t \rightarrow \infty} \min _{\theta(t) \leq s \leq t}\left|z_{0}(s)\right|=0
$$

Proof. By the use of Theorem 3.3 we observe that there is a $t_{2} \geq T$ and a solution $z_{0}(t)$ of (1.2) with property

$$
\left|z_{0}(t)\right| \leq \frac{C}{|\widetilde{\gamma}(t)|-|\widetilde{c}(t)|} \exp \left[\int_{T}^{t} \xi(s) d s\right]
$$

for $t \geq t_{2}$. Suppose that (3.46) is not satisfied. Then, there is $\varepsilon_{0}>0$ such that

$$
\limsup _{t \rightarrow \infty} \min _{\theta(t) \leq s \leq t}\left|z_{0}(s)\right|>\varepsilon_{0}
$$

Choose $N \in \mathbb{N}$ such that

$$
\max \left\{R_{N}, \frac{2}{\mu} R_{N} e^{-v}\right\}<\varepsilon_{0}
$$

It holds that

$$
\min _{\theta(\tau) \leq s \leq \tau}\left|z_{0}(s)\right|>\max \left\{R_{N}, \frac{2}{\mu} R_{N} e^{-v}\right\}
$$


for some $\tau>\max \left\{T, \tau_{N}, t_{2}\right\}$. In view of (3.44), we can suppose that

$$
\left|\widetilde{\beta}_{N}(\tau)\right| C \max _{\theta(\tau) \leq s \leq \tau} \frac{\exp \left[\int_{T}^{s} \xi(\sigma) d \sigma\right]}{|\widetilde{\gamma}(s)|-|\widetilde{c}(s)|} \sum_{k=1}^{m} \int_{\theta_{k}(\tau)}^{\tau}(|\widetilde{\gamma}(s)|+|\widetilde{c}(s)|) d s<\frac{1}{2} R_{N} e^{-\nu}
$$

Therefore, taking into account (2.5), (3.47), (3.50), and (3.51) and the nonpositiveness of $\beta_{N}$, we have

$$
\begin{aligned}
(|\tilde{\gamma}(\tau)| & -|\widetilde{c}(\tau)|)\left|z_{0}(\tau)\right|+\tilde{\beta}_{N}(\tau) \max _{\theta(\tau) \leq s \leq \tau}\left|z_{0}(s)\right| \sum_{k=1}^{m} \int_{\theta_{k}(\tau)}^{\tau}(|\tilde{\gamma}(s)|+|\widetilde{c}(s)|) d s \\
\geq & (|\tilde{\gamma}(\tau)|-|\widetilde{c}(\tau)|)\left|z_{0}(\tau)\right| \\
& +\tilde{\beta}_{N}(\tau) C \max _{\theta(\tau) \leq s \leq \tau} \frac{\exp \left[\int_{T}^{s} \xi(\sigma) d \sigma\right]}{|\tilde{\gamma}(s)|-|\widetilde{c}(s)|} \sum_{k=1}^{m} \int_{\theta_{k}(\tau)}^{\tau}(|\tilde{\gamma}(s)|+|\widetilde{c}(s)|) d s \\
\geq & \mu \frac{2}{\mu} R_{N} e^{-v}-\frac{1}{2} R_{N} e^{-v}>R_{N} e^{-v} .
\end{aligned}
$$

Moreover, (3.45) implies that

$$
\inf _{\tau \leq t<\infty}\left[\int_{\tau}^{t} \tilde{\Lambda}_{N}(s) d s-\ln (|\tilde{\gamma}(t)|+|\widetilde{c}(t)|)\right] \geq v>-\infty
$$

By Theorem 3.1, we obtain an estimation

$$
\left|z_{0}(t)\right| \geq \frac{\Psi(\tau)}{|\tilde{\gamma}(t)|+|\tilde{c}(t)|} \exp \left[\int_{\tau}^{t} \tilde{\Lambda}_{N}(s) d s\right]
$$

for all $t \geq \tau, \Psi$ being defined by

$$
\Psi(\tau)=(|\widetilde{\gamma}(\tau)|-|\widetilde{c}(\tau)|)\left|z_{0}(\tau)\right|+\tilde{\beta}_{N}(\tau) \max _{\theta(\tau) \leq s \leq \tau}\left|z_{0}(s)\right| \sum_{k=1}^{m} \int_{\theta_{k}(\tau)}^{\tau}(|\tilde{\gamma}(s)|+|\widetilde{c}(s)|) d s .
$$

Relation (3.47) together with (3.54) yields

$$
\frac{\Psi(\tau)}{|\tilde{\gamma}(t)|+|\widetilde{c}(t)|} \exp \left[\int_{\tau}^{t} \tilde{\Lambda}_{N}(s) d s\right] \leq \frac{C}{|\widetilde{\gamma}(t)|-|\widetilde{c}(t)|} \exp \left[\int_{T}^{t} \xi(s) d s\right]
$$

that is

$$
\int_{T}^{t}\left[\tilde{\Lambda}_{N}(s)-\xi(s)\right] d s+\ln \frac{|\widetilde{\gamma}(t)|-|\widetilde{c}(t)|}{|\widetilde{\gamma}(t)|+|\widetilde{c}(t)|} \leq \int_{T}^{\tau} \tilde{\Lambda}_{N}(s) d s-\ln \left[C^{-1} \Psi(\tau)\right]
$$

for $t \geq \tau$. However, the last inequality contradicts (3.43) and Theorem 3.4 is proved. 
From Theorem 3.1 we easily obtain several corollaries.

Corollary 3.5. Let the assumptions of Theorem 3.1 be fulfilled with $R_{0}>0$. If

$$
\liminf _{t \rightarrow \infty}\left[\int_{t_{1}}^{t} \tilde{\Lambda}_{0}(s) d s-\ln (|\tilde{\gamma}(t)|+|\widetilde{c}(t)|)\right]=\varsigma>v
$$

then for any $\varepsilon, 0<\varepsilon<R_{0} e^{s^{-v}}$, there is $t_{2} \geq t_{1}$ such that

$$
|z(t)|>\varepsilon
$$

for all $t \geq t_{2}$, for which $z(t)$ is defined.

Proof. Without loss of generality we can assume that $\varepsilon>R_{0}$. Choose $x, 0<x<1$ such that $R_{0}<\varepsilon<\chi R_{0} e^{\varsigma^{-\nu}}$. In view of (3.58), there is $t_{2} \geq t_{1}$ such that

$$
\int_{t_{1}}^{t} \tilde{\Lambda}_{0}(s) d s-\ln (|\tilde{\gamma}(t)|+|\widetilde{c}(t)|)>s+\ln x
$$

for $t \geq t_{2}$. Hence,

$$
\int_{t_{1}}^{t} \tilde{\Lambda}_{0}(s) d s-\ln (|\tilde{\gamma}(t)|+|\widetilde{c}(t)|)>v+\ln \frac{\varepsilon}{R_{0}}
$$

for $t \geq t_{2}$. Estimation (3.4) together with (3.2) now yields

$$
|z(t)|>R_{0} e^{-v} e^{v} \frac{\varepsilon}{R_{0}}=\varepsilon
$$

for all $t \geq t_{2}$, for which $z(t)$ is defined.

Corollary 3.6. Let the assumptions of Theorem 3.1 be fulfilled with $R_{0}>0$. If

$$
\lim _{t \rightarrow \infty}\left[\int_{t_{1}}^{t} \tilde{\Lambda}_{0}(s) d s-\ln (|\tilde{\gamma}(t)|+|\widetilde{c}(t)|)\right]=\infty
$$

then for any $\varepsilon>0$ there exists $t_{2} \geq t_{1}$ such that (3.59) holds for all $t \geq t_{2}$, for which $z(t)$ is defined. example.

The efficiency of Theorem 3.1 and Corollary 3.6 is demonstrated in the following Example 3.7. Consider (1.2) where $a(t) \equiv 4+3 i, b(t) \equiv 2, A_{k}(t) \equiv 0, B_{k}(t) \equiv 0$ for $k=1, \ldots, m$, $\theta_{k}(t)=t+(1 / 2 k)(\cos k t-1), g\left(t, z, w_{1}, \ldots, w_{m}\right)=3 z+\sum_{k=1}^{m}(1 / 2 m) e^{-t} w_{k}$. 
Obviously, $t-(1 / k) \leq \theta_{k}(t) \leq t$ and $1 / 2 \leq \theta_{k}^{\prime}(t) \leq(3 / 2)$. Suppose that $t_{0}=1$ and $T \geq 2$. Then, $\tilde{\gamma} \equiv 3+\sqrt{5}, \tilde{c} \equiv-2 i$. Further,

$$
\begin{aligned}
& \left|\tilde{\gamma}(t) g\left(t, z, w_{1}, \ldots, w_{m}\right)+\tilde{c}(t) \bar{g}\left(t, z, w_{1}, \ldots, w_{m}\right)\right| \leq 3|\tilde{\gamma}(t) z+\widetilde{c}(t) \bar{z}| \\
& \quad+\sum_{k=1}^{m} \frac{1}{2 m} \mathrm{e}^{-t}\left|\widetilde{\gamma}\left(\theta_{k}(t)\right) w_{k}+\widetilde{c}\left(\theta_{k}(t)\right) \bar{w}_{k}\right| .
\end{aligned}
$$

Taking $\tilde{\varkappa}_{0}(t) \equiv 3, \widetilde{\kappa}_{0 k}(t)=(1 / 2 m) \mathrm{e}^{-t}, \tau_{0}=T, R_{0}=0, \tilde{\vartheta}(t) \equiv 0, \tilde{\beta}_{0}(t)=-(1 / m) \mathrm{e}^{-t}$, $\tilde{\Lambda}_{0}(t)=\tilde{\Theta}_{0}(t)=1-\mathrm{e}^{-t}(>0)$ in Theorem 3.1, we have

$$
\theta_{k}^{\prime}(t) \tilde{\beta}_{0}(t) \leq-\tilde{\lambda}_{0 k}(t), \quad \tilde{\beta}_{0}^{\prime}(t) \geq \tilde{\Theta}_{0}(t) \tilde{\beta}_{0}(t)
$$

for $t \in[T, \infty)$ and Theorem 3.1 and Corollary 3.6 are applicable to the considered equation.

As a corollary of Theorem 3.3 we obtain sufficient conditions for the existence of a bounded solution of (1.2) or the existence of a solution $z_{0}(t)$ of (1.2) satisfying $\lim _{t \rightarrow \infty} z_{0}(t)=$ 0 .

Corollary 3.8. Let the assumptions of Theorem 3.3 be satisfied. If

$$
\limsup _{t \rightarrow \infty}\left[\frac{1}{|\tilde{\gamma}(t)|-|\tilde{c}(t)|} \exp \left(\int_{T}^{t} \xi(s) d s\right)\right]<\infty,
$$

then there is a bounded solution $z_{0}(t)$ of (1.2). If

$$
\lim _{t \rightarrow \infty}\left[\frac{1}{|\tilde{\gamma}(t)|-|\widetilde{c}(t)|} \exp \left(\int_{T}^{t} \xi(s) d s\right)\right]=0,
$$

then there is a solution $z_{0}(t)$ of $(1.2)$ such that

$$
\lim _{t \rightarrow \infty} z_{0}(t)=0
$$

The next example shows how Theorem 3.3 and Corollary 3.8 (namely the first part) can be used.

Example 3.9. Consider (1.2) where $a(t) \equiv 4+3 i, b(t) \equiv i, A_{k}(t) \equiv 0, B_{k}(t) \equiv 0, \theta_{k}(t)=t-e^{-k t}$ for $k=1, \ldots, m, g\left(t, z, w_{1}, \ldots, w_{m}\right)=(1 / 2) z+\sum_{k=1}^{m}(1 / 4 m) w_{k}+e^{-t}$.

Obviously $t-1 \leq \theta_{k}(t) \leq t$ and $\theta_{k}^{\prime}(t)=1+k \mathrm{e}^{-k t} \geq 1>0$ for $t \geq 0$. Suppose that $t_{0}=1$ and $T \geq 2$. Then,

$$
\begin{gathered}
\tilde{\gamma}(t)=\operatorname{Im} a(t)+\sqrt{(\operatorname{Im} a(t))^{2}-|b(t)|^{2}} \operatorname{sgn}(\operatorname{Im} a(t)) \equiv 3+2 \sqrt{2}, \\
\tilde{c}(t)=-i b(t) \equiv 1 .
\end{gathered}
$$


Further,

$$
\begin{aligned}
& \left|\tilde{\gamma}(t) g\left(t, z, w_{1}, \ldots, w_{m}\right)+\widetilde{c}(t) \bar{g}\left(t, z, w_{1}, \ldots, w_{m}\right)\right| \\
& \leq \frac{|\widetilde{\gamma}|+|\widetilde{c}|}{|\widetilde{\gamma}|-|\widetilde{c}|} \frac{1}{2}|\widetilde{\gamma}(t) z+\widetilde{c}(t) \bar{z}| \\
& +\frac{|\tilde{r}|+|\widetilde{c}|}{|\widetilde{\gamma}|-|\widetilde{c}|} \sum_{k=1}^{m}\left[\frac{1}{4 m}\left|\tilde{\gamma}\left(\theta_{k}(t)\right) w_{k}+\widetilde{c}\left(\theta_{k}(t)\right) \bar{w}_{k}\right|\right]+\mathrm{e}^{-t} \\
& =\frac{\sqrt{2}}{2}|\tilde{\gamma}(t) z+\tilde{c}(t) \bar{z}|+\sqrt{2} \sum_{k=1}^{m}\left[\frac{1}{4 m}\left|\tilde{\gamma}\left(\theta_{k}(t)\right) w_{k}+\tilde{c}\left(\theta_{k}(t)\right) \bar{w}_{k}\right|\right]+\mathrm{e}^{-t} .
\end{aligned}
$$

If we take $\tilde{\varkappa}(t) \equiv \sqrt{2} / 2, \widetilde{\kappa}_{k}(t)=\sqrt{2} / 4 m, \tilde{\vartheta}(t) \equiv 0, \tilde{\beta}(t)=-\sqrt{2} / 4 m, \tilde{\Lambda}(t)=\tilde{\Theta}(t)=$ $4-(\sqrt{2} / 2)$ in Theorem 3.3, we observe that

$$
\theta_{k}^{\prime}(t) \tilde{\beta}(t)=-\left(1+k^{-k t} \mathrm{e}\right) \frac{\sqrt{2}}{4 m} \leq-\frac{\sqrt{2}}{4 m}=-\tilde{\lambda}_{k}(t)
$$

for $t \in[T, \infty)$. Then, for $\xi \equiv 0$ and $C=1$, we have

$$
\begin{aligned}
\tilde{\Lambda}(t) & +\tilde{\beta}(t) \sum_{k=1}^{m} \theta_{k}^{\prime}(t) \exp \left[-\int_{\theta_{k}(t)}^{t} \xi(s) d s\right]-\xi(t) \\
& =4-\frac{\sqrt{2}}{2}-\frac{\sqrt{2}}{4 m} \sum_{k=1}^{m}\left(1+k^{-k t} \mathrm{e}^{m}\right) \geq 4-\frac{\sqrt{2}}{2}-\frac{\sqrt{2}}{4 m} \cdot 2 m=4-\sqrt{2}>1 \\
& >\mathrm{e}=\rho(t) C^{-1} \exp \left(-\int_{T}^{t} \xi(s) d s\right)
\end{aligned}
$$

for $t \in[T, \infty)$ and

$$
\limsup _{t \rightarrow \infty}\left[\frac{1}{|\widetilde{\gamma}(t)|-|\widetilde{c}(t)|} \exp \left(\int_{T}^{t} \xi(s) d s\right)\right]=\frac{1}{2+2 \sqrt{2}}<\infty,
$$

and hence the assertions of Theorem 3.3 and the first part of Corollary 3.8 hold true.

\section{Summary}

We investigated the problem of instability and asymptotic behaviour of real two-dimensional differential system with a finite number of nonconstant delays. We focused on the case corresponding to the situation when the equilibrium 0 of the autonomous system (2.2) is a focus or a centre and it is unstable. We obtained several criteria for instability properties of the solutions as well as conditions for the existence of bounded solutions. We used the methods of complexification, the method of Lyapunov-Krasovskii functional and a Ważewski topological principle for retarded functional differential equations of Carathéodory type. At the end we supplied several corollaries and explanatory examples. 


\title{
Acknowledgment
}

\author{
Z. Šmarda was supported by Project FEKT-S-11-2(921).
}

\section{References}

[1] J. Baštinec, L. Berezansky, J. Diblík, and Z. Šmarda, “On the critical case in oscillation for differential equations with a single delay and with several delays," Abstract and Applied Analysis, vol. 2010, Article ID 417869, 20 pages, 2010.

[2] J. Čermák, "On matrix differential equations with several unbounded delays," European Journal of Applied Mathematics, vol. 17, no. 4, pp. 417-433, 2006.

[3] J. Čermák and S. Dvořáková, "Asymptotic estimation for some nonlinear delay differential equations," Results in Mathematics, vol. 51, no. 3-4, pp. 201-213, 2008.

[4] J. Diblík, "Asymptotic representation of solutions of equation $\dot{y}(t)=\beta(t)[y(t)-y(t-\tau(t))]$," Journal of Mathematical Analysis and Applications, vol. 217, no. 1, pp. 200-215, 1998.

[5] J. Diblík, "A criterion for existence of positive solutions of systems of retarded functional-differential equations," Nonlinear Analysis, vol. 38, no. 3, pp. 327-339, 1999.

[6] J. Diblík and M. Rủžičková, "Exponential solutions of equation $\dot{y}(t)=\beta(t)[y(t-\delta)-y(t-\tau)]$," Journal of Mathematical Analysis and Applications, vol. 294, no. 1, pp. 273-287, 2004.

[7] J. Diblík and Z. Svoboda, "Positive solutions of retarded functional differential equations," Nonlinear Analysis, vol. 63, no. 5-7, pp. e813-e821, 2005.

[8] J. Diblík and Z. Svoboda, "Positive solutions of p-type retarded functional differential equations," Nonlinear Analysis, vol. 64, no. 8, pp. 1831-1848, 2006.

[9] J. Diblík, Z. Svoboda, and Z. Šmarda, "Explicit criteria for the existence of positive solutions for a scalar differential equation with variable delay in the critical case," Computers $\mathcal{E}$ Mathematics with Applications, vol. 56, no. 2, pp. 556-564, 2008.

[10] J. Diblík, Z. Svoboda, and Z. Šmarda, "Retract principle for neutral functional differential equations," Nonlinear Analysis, vol. 71, no. 12, pp. e1393-e1400, 2009.

[11] I. Györi and M. Pituk, "Stability criteria for linear delay differential equations," Differential and Integral Equations, vol. 10, no. 5, pp. 841-852, 1997.

[12] M. Pituk, "Asymptotic behavior and oscillation of functional differential equations," Journal of Mathematical Analysis and Applications, vol. 322, no. 2, pp. 1140-1158, 2006.

[13] E. Špániková and H. Šamajová, "Asymptotic properties of solutions to n-dimensional neutral differential systems," Nonlinear Analysis, vol. 71, no. 7-8, pp. 2877-2885, 2009.

[14] K. P. Rybakowski, "Ważewski's principle for retarded functional differential equations," Journal of Differential Equations, vol. 36, no. 1, pp. 117-138, 1980.

[15] K. P. Rybakowski, "A topological principle for retarded functional-differential equations of Carathéodory type," Journal of Differential Equations, vol. 39, no. 2, pp. 131-150, 1981.

[16] M. Ráb and J. Kalas, "Stability of dynamical systems in the plane," Differential and Integral Equations, vol. 3, no. 1, pp. 127-144, 1990.

[17] J. Kalas and L. Baráková, "Stability and asymptotic behaviour of a two-dimensional differential system with delay," Journal of Mathematical Analysis and Applications, vol. 269, no. 1, pp. 278-300, 2002.

[18] J. Kalas and J. Osička, "Bounded solutions of dynamical systems in the plane under the conditions of instability," Mathematische Nachrichten, vol. 170, pp. 133-147, 1994.

[19] J. Kalas, "Asymptotic behaviour of a two-dimensional differential system with delay under the conditions of instability," Nonlinear Analysis, vol. 62, no. 2, pp. 207-224, 2005.

[20] J. Rebenda, "Asymptotic properties of solutions of real two-dimensional differential systems with a finite number of constant delays," Memoirs on Differential Equations and Mathematical Physics, vol. 41, pp. 97-114, 2007. 


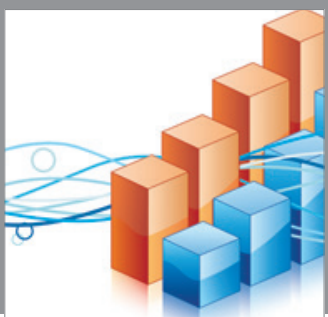

Advances in

Operations Research

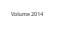

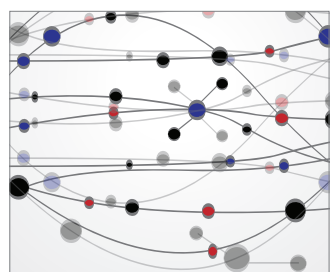

\section{The Scientific} World Journal
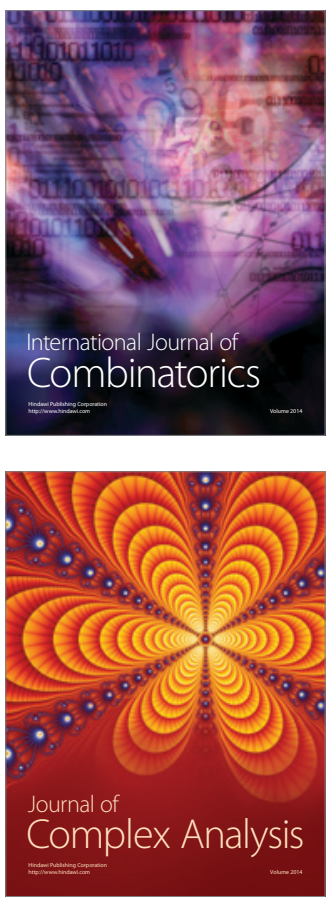

International Journal of

Mathematics and

Mathematical

Sciences
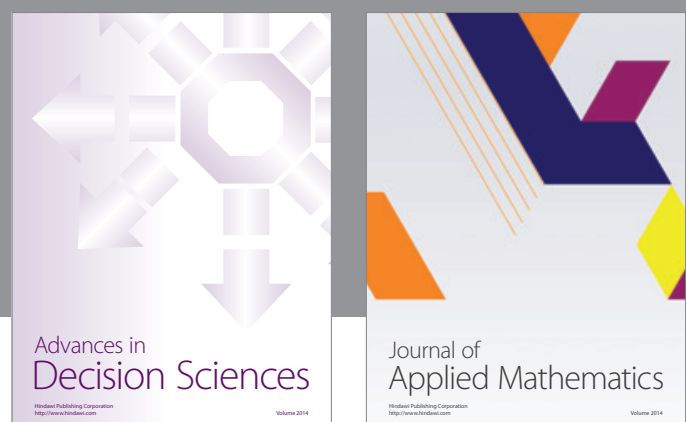

Journal of

Applied Mathematics
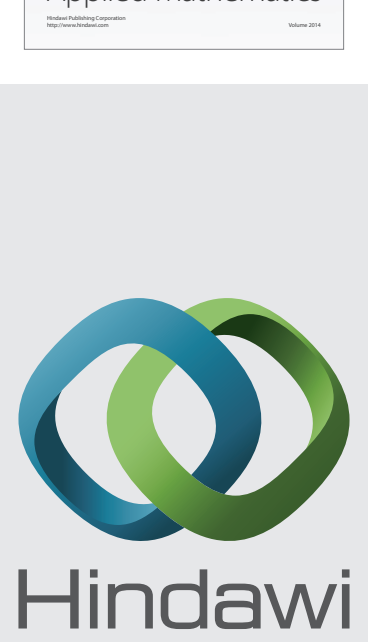

Submit your manuscripts at http://www.hindawi.com
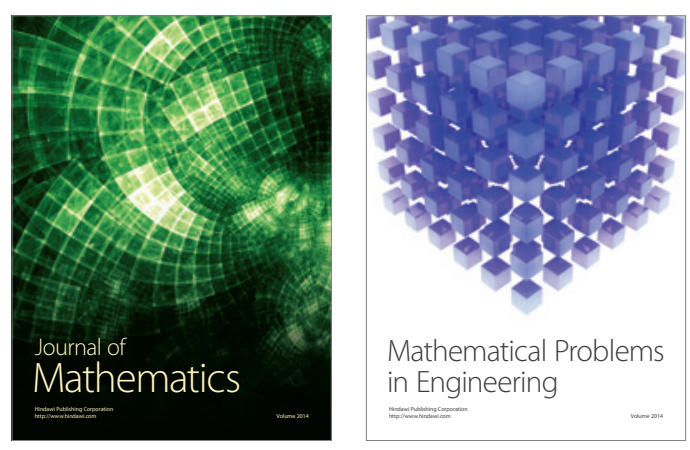

Mathematical Problems in Engineering
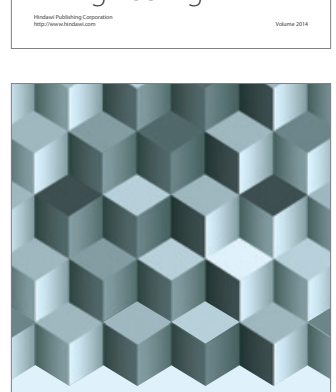

Journal of

Function Spaces
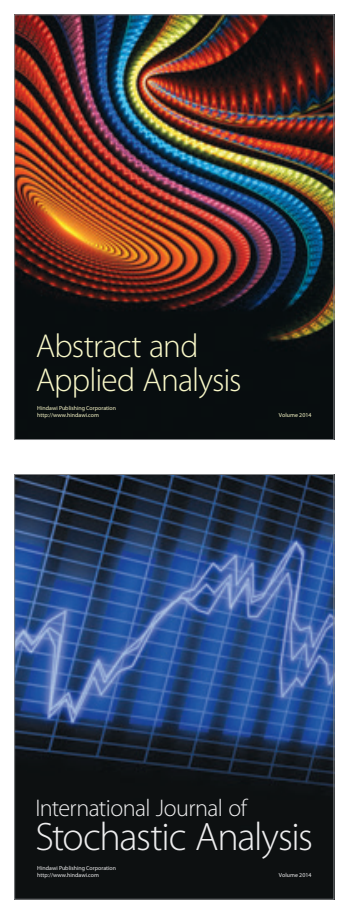

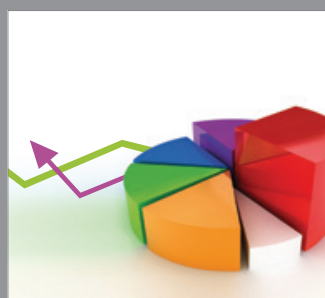

ournal of

Probability and Statistics

Promensencen
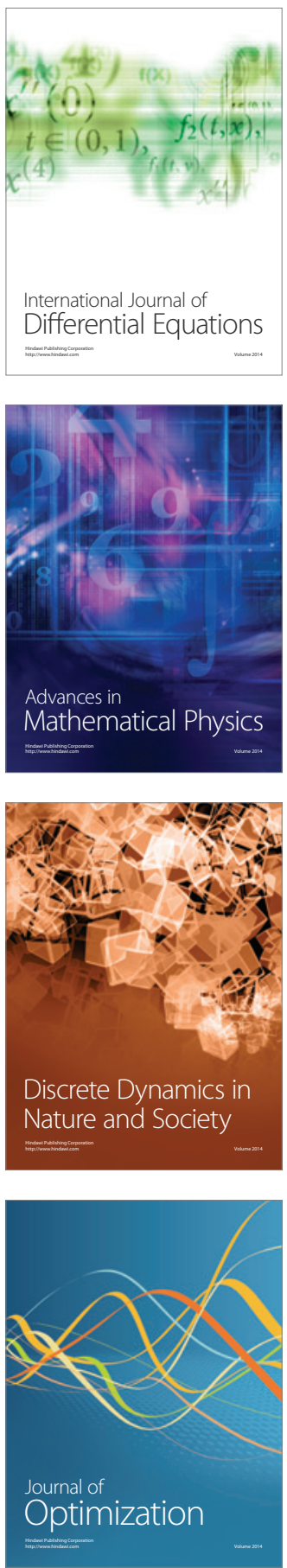